\title{
On Solvability of Third-Order Operator Differential Equation with Parabolic Principal Part in Weighted Space
}

\author{
Araz R. Aliev, ${ }^{1,2}$ Sabir S. Mirzoev, ${ }^{2,3}$ and Mustafa A. Soylemezo ${ }^{4}$ \\ ${ }^{1}$ Azerbaijan State Oil and Industry University, 1010 Baku, Azerbaijan \\ ${ }^{2}$ Institute of Mathematics and Mechanics of ANAS, 1141 Baku, Azerbaijan \\ ${ }^{3}$ Baku State University, 1148 Baku, Azerbaijan \\ ${ }^{4}$ Baku Engineering University, Khirdalan City, 0101 Baku, Azerbaijan \\ Correspondence should be addressed to Sabir S. Mirzoev; mirzoyevsabir@mail.ru
}

Received 30 April 2017; Accepted 27 September 2017; Published 31 October 2017

Academic Editor: Hugo Leiva

Copyright (c) 2017 Araz R. Aliev et al. This is an open access article distributed under the Creative Commons Attribution License, which permits unrestricted use, distribution, and reproduction in any medium, provided the original work is properly cited.

\begin{abstract}
Sufficient conditions are found for the correct and unique solvability of a class of third-order parabolic operator differential equations, whose principal parts have multiple characteristics, in a Sobolev-type space with exponential weight. The estimates for the norms of intermediate derivative operators are obtained and the relationship between these estimates and solvability conditions is established. Besides, the connection is found between the order of exponential weight and the lower bound for the spectrum of abstract operator appearing in the principal part of the equation.
\end{abstract}

\section{Problem Statement}

Let $H$ be a separable Hilbert space with the scalar product $(x, y), x, y \in H$, and let $A$ be a self-adjoint positive definite operator in $H\left(A=A^{*} \geq c E, c>0, E\right.$ is a unit operator). Denote by $H_{\gamma}(\gamma \geq 0)$ a scale of Hilbert spaces generated by the operator $A$; that is, $H_{\gamma}=\operatorname{Dom}\left(A^{\gamma}\right),(x, y)_{\gamma}=\left(A^{\gamma} x, A^{\gamma} y\right)$, $x, y \in \operatorname{Dom}\left(A^{\gamma}\right)$. For $\gamma=0$, we assume that $H_{0}=H,(x, y)_{0}=$ $(x, y), x, y \in H$.

Denote by $L_{2}(R ; H)(R=(-\infty,+\infty))$ a space of measurable (see [1]) functions with the values in $H$ equipped with the norm

$$
\|g\|_{L_{2}(R ; H)}=\left(\int_{-\infty}^{+\infty}\|g(t)\|^{2} d t\right)^{1 / 2}
$$

and by $W_{2}^{3}(R ; H)$ a space of functions with the values in $H$ such that $d^{3} v(t) / d t^{3}, A^{3} v(t) \in L_{2}(R ; H)$, equipped with the norm

$$
\|v\|_{W_{2}^{3}(R ; H)}=\left(\left\|\frac{d^{3} v}{d t^{3}}\right\|_{L_{2}(R ; H)}^{2}+\left\|A^{3} v\right\|_{L_{2}(R ; H)}^{2}\right)^{1 / 2} .
$$

For more details on the space $W_{2}^{3}(R ; H)$ see $[2$, Chapter 1$]$.
Note that throughout this paper all the derivatives are understood in the sense of the theory of distributions, and the operator $A^{\gamma}$ is defined by the spectral decomposition of the operator $A$; that is, $A^{\gamma}=\int_{c}^{+\infty} \sigma^{\gamma} d E_{\sigma}, \quad \gamma \geq 0$, where $E_{\sigma}$ is a decomposition of the unit of the operator $A$.

Now, let us recall one fact related to the space $W_{2}^{3}(R ; H)$.

It is known that if $v(t) \in W_{2}^{3}(R ; H)$, then the inequalities

$$
\left\|A^{3-j} \frac{d^{j} v}{d t^{j}}\right\|_{L_{2}(R ; H)} \leq c_{j}\|v\|_{W_{2}^{3}(R ; H)}, \quad j=\overline{0,3},
$$

are valid, where $c_{j}, j=\overline{0,3}$, are constants independent of function $v(t)$. This fact is referred to as the intermediate derivatives theorem (see [2, Chapter 1]). Also, these inequalities are usually referred to as Kolmogorov-type inequalities.

Let $-\infty<\kappa<+\infty$. For the functions $u(t)$ defined on $R$ with the values in $H$, we introduce the following spaces with the weight $e^{-(\kappa / 2) t}$ :

$$
\begin{aligned}
& L_{2, \kappa}(R ; H)=\left\{u(t):\|u\|_{L_{2, \kappa}(R ; H)}\right. \\
& \left.\quad=\left(\int_{-\infty}^{+\infty}\|u(t)\|_{H}^{2} e^{-\kappa t} d t\right)^{1 / 2}<+\infty\right\},
\end{aligned}
$$




$$
\begin{aligned}
& W_{2, \kappa}^{3}(R ; H)=\left\{u(t):\|u\|_{W_{2, \kappa}^{3}(R ; H)}\right. \\
& \quad=\left(\int_{-\infty}^{+\infty}\left(\left\|\frac{d^{3} u(t)}{d t^{3}}\right\|_{H}^{2}+\left\|A^{3} u(t)\right\|_{H}^{2}\right) e^{-\kappa t} d t\right)^{1 / 2} \\
& \quad<+\infty\} .
\end{aligned}
$$

Obviously, in case $\kappa=0$ we get the spaces $L_{2,0}(R ; H)=$ $L_{2}(R ; H), W_{2,0}^{3}(R ; H)=W_{2}^{3}(R ; H)$.

In the sequel, by $L(X, Y)$ we will mean a set of linear bounded operators from the Hilbert space $X$ to another Hilbert space $Y$. If $Y=X$, we will write $L(X)$ instead of $L(X, Y)$. By $\sigma(A)$ we will denote the spectrum of the operator A.

Consider the operator differential equation

$$
\begin{array}{r}
\left(\frac{d}{d t}+A\right)^{3} u(t)+A_{1} \frac{d^{2} u(t)}{d t^{2}}+A_{2} \frac{d u(t)}{d t}=f(t) \\
t \in R
\end{array}
$$

where $A=A^{*} \geq c E, c>0, A_{1}, A_{2}$ are linear and, in general, unbounded operators, $f(t) \in L_{2, \kappa}(R ; H), u(t) \in W_{2, \kappa}^{3}(R ; H)$.

Definition 1. If for every $f(t) \in L_{2, \kappa}(R ; H)$ there exists a vector function $u(t) \in W_{2, \kappa}^{3}(R ; H)$ which satisfies (5) almost everywhere with

$$
\|u\|_{W_{2, \kappa}^{3}(R ; H)} \leq \text { const }\|f\|_{L_{2, \kappa}(R ; H)},
$$

then this vector function is called a regular solution of (5), and (5) is said to be regularly solvable.

The principal part of (5) has multiple characteristics, so, according to the classification of [3], this equation belongs to the class of parabolic operator differential equations. The equations of form (5) characterize the problems of diffusion or heat conductivity in viscoelastic media [4]. Besides, such equations are also interesting in view of the fact that some classes of equations, which can be useful in modeling the problems of world population growth, can be reduced to them [5].

Note that the solvability issues for the operator differential equations in the spaces without weights have been studied quite widely (see Krein [6], Lions and Magenes [2], V. I. Gorbachuk and M. L. Gorbachuk [7], Goldstein [8], Yakubov $[9,10]$, S. Yakubov and Y. Yakubov [11], and the references therein). Fundamental contribution to this field was made by Gasymov in [12-14]. These issues remain as relevant today as they were long before. A lot of researches appeared in this field over the last years; see, for example, Aliev [15], Aliev and Mirzoev [16], Favini et al. [17], Favini and Yakubov [18, 19], Favini et al. [20], Mirzoev et al. [21, 22], Aliev and Yakubov $[23,24]$, and Mirzoev et al. [25].
Despite the number of works covering the solvability of operator differential equations in weighted spaces being relatively small (see, e.g., Dubinskiu [3], Shkalikov [26], Mirzoev [27], and Aliev [28]), recently a lot of works appeared dedicated to the issues of regular and normal solvability in weighted spaces for operator differential equations with multiple characteristics (see Aliev [29], Mirzoev and Humbataliev [30], Aliev and Elbably [31], and Aliev and Lachinova [32]). But the equations considered in the abovementioned works, except for [32], belong to the class of quasielliptic operator differential equations [3]. In [32], the initialboundary value problem was studied for parabolic equation on the half-axis $R_{+}=[0,+\infty)$.

In this work, we find the regular solvability conditions for (5) on the entire axis. We also obtain the estimates for the norms of intermediate derivative operators in a Sobolevtype space through the norm of the operator generated by the principal part of (5) (it should be noted here that the estimates for the norms of intermediate derivatives for scalar functions have been obtained in $[33,34]$ and the references therein). Moreover, we establish the relationship between these estimates and the regular solvability conditions for (5). The found regular solvability conditions are expressed in terms of operator coefficients of (5), which makes them easily verifiable and convenient for use both in theoretical problems and in applications.

\section{Solvability of (5) in $W_{2, \kappa}^{3}(R ; H)$ \\ When $A_{j}=0, j=1,2$}

We first consider (5) in case $A_{1}=A_{2}=0$.

Denote by $P_{0}$ the operator acting from $W_{2, \kappa}^{3}(R ; H)$ to $L_{2, \kappa}(R ; H)$ as follows:

$$
P_{0} u(t) \equiv\left(\frac{d}{d t}+A\right)^{3} u(t), \quad u(t) \in W_{2}^{3}(R ; H) .
$$

The following theorem is true.

Theorem 2. Let $A$ be a self-adjoint positive definite operator with the lower bound for its spectrum $\lambda_{0}\left(A=A^{*} \geq\right.$ $\left.\lambda_{0} E, \lambda_{0}>0\right)$ and $\kappa>-2 \lambda_{0}$. Then the operator $P_{0}$ performs an isomorphism between the spaces $W_{2, \kappa}^{3}(R ; H)$ and $L_{2, \kappa}(R ; H)$.

Proof. Let us make a change $u(t)=v(t) e^{(\kappa / 2) t}$ in the equation

$$
P_{0} u(t)=f(t)
$$

$u(t) \in W_{2, \kappa}^{3}(R ; H), f(t) \in L_{2, \kappa}(R ; H)$. Then $v(t)=$ $u(t) e^{-(\kappa / 2) t} \in W_{2}^{3}(R ; H)$. As

$$
\left(\frac{d}{d t}+A\right)^{3} u(t)=e^{(\kappa / 2) t}\left(\frac{d}{d t}+\frac{\kappa}{2}+A\right)^{3} v(t)=f(t),
$$

we have

$$
\left(\frac{d}{d t}+\frac{\kappa}{2}+A\right)^{3} v(t)=f(t) e^{-(\kappa / 2) t}
$$


Taking into account the fact that $g(t)=f(t) e^{-(\kappa / 2) t} \in$ $L_{2}(R ; H)$, we can rewrite (10) in the form

$$
\left(\frac{d}{d t}+\frac{\kappa}{2}+A\right)^{3} v(t)=g(t)
$$

in the space $L_{2}(R ; H)$; that is, $v(t) \in W_{2}^{3}(R ; H), g(t) \in$ $L_{2}(R ; H)$.

Denote

$$
P_{0, \kappa} v(t)=\left(\frac{d}{d t}+\frac{\kappa}{2}+A\right)^{3} v(t), \quad v(t) \in W_{2}^{3}(R ; H) .
$$

Then (11) can be rewritten as follows:

$$
P_{0, \kappa} v(t)=g(t),
$$

where $v(t) \in W_{2}^{3}(R ; H), g(t) \in L_{2}(R ; H)$. To solve the last equation, we make use of the Fourier transform:

$$
\left(\left(i \xi+\frac{\kappa}{2}\right) E+A\right)^{3} \widehat{v}(\xi)=\widehat{g}(\xi),
$$

where $\widehat{v}(\xi), \widehat{g}(\xi)$ are Fourier transforms of the vector functions $v(t), g(t)$, respectively. Let us show that for $\kappa>-2 \lambda_{0}$ the operator pencil

$$
P_{0, \kappa}(i \xi ; A)=\left(\left(i \xi+\frac{\kappa}{2}\right) E+A\right)^{3}
$$

is invertible. In fact, let $\lambda \in \sigma(A)\left(\lambda \geq \lambda_{0}\right)$. Then the characteristic polynomial (15) has the form

$$
P_{0, \kappa}(i \xi ; \lambda)=\left(i \xi+\frac{\kappa}{2}+\lambda\right)^{3} .
$$

Hence we have

$$
\begin{aligned}
\left|P_{0, \kappa}(i \xi ; \lambda)\right| & =\left|\left(i \xi+\frac{\kappa}{2}+\lambda\right)^{3}\right|=\left(\left(\lambda+\frac{\kappa}{2}\right)^{2}+\xi^{2}\right)^{3 / 2} \\
& \geq\left(\lambda+\frac{\kappa}{2}\right)^{3} \geq\left(\lambda_{0}+\frac{\kappa}{2}\right)^{3}>0, \quad \xi \in R,
\end{aligned}
$$

that is, the spectral decomposition of the operator $A$ implies the invertibility of the operator pencil $P_{0, \kappa}(i \xi ; A)$ for $\kappa>$ $-2 \lambda_{0}$. Consequently, from (14) we can find $\widehat{v}(\xi)$ :

$$
\widehat{v}(\xi)=\left(\left(i \xi+\frac{\kappa}{2}\right) E+A\right)^{-3} \widehat{g}(\xi) .
$$

Thus,

$$
v(t)=\frac{1}{\sqrt{2 \pi}} \int_{-\infty}^{+\infty}\left(\left(i \xi+\frac{\kappa}{2}\right) E+A\right)^{-3} \hat{g}(\xi) e^{i \xi t} d \xi .
$$

It is clear that $v(t)$ satisfies (11) almost everywhere.

Let us prove that $v(t) \in W_{2}^{3}(R ; H)$. In fact, by virtue of well-known Plancherel theorem, it suffices to show that $A^{3} \widehat{v}(\xi) \in L_{2}(R ; H)$ and $-i \xi^{3} \widehat{v}(\xi) \in L_{2}(R ; H)$. Obviously,

$$
\begin{aligned}
\|v\|_{W_{2}^{3}(R ; H)}^{2} & =\left\|\frac{d^{3} v}{d t^{3}}\right\|_{L_{2}(R ; H)}^{2}+\left\|A^{3} v\right\|_{L_{2}(R ; H)}^{2} \\
& =\left\|-i \xi^{3} \widehat{v}(\xi)\right\|_{L_{2}(R ; H)}^{2}+\left\|A^{3} \widehat{v}(\xi)\right\|_{L_{2}(R ; H)}^{2} .
\end{aligned}
$$

As

$$
\begin{aligned}
& \left\|A^{3} \widehat{v}(\xi)\right\|_{L_{2}(R ; H)} \\
& =\left\|A^{3}\left(\left(i \xi+\frac{\kappa}{2}\right) E+A\right)^{-3} \widehat{g}(\xi)\right\|_{L_{2}(R ; H)} \\
& \leq \sup _{\xi \in R}\left\|A^{3}\left(\left(i \xi+\frac{\kappa}{2}\right) E+A\right)^{-3}\right\|_{H \rightarrow H}\|\widehat{g}(\xi)\|_{L_{2}(R ; H)},
\end{aligned}
$$

let us estimate the norm $\left\|A^{3}((i \xi+\kappa / 2) E+A)^{-3}\right\|_{H \rightarrow H}$ for $\xi \epsilon$ $R$. Spectral theory of self-adjoint operators implies

$$
\begin{aligned}
& \left\|A^{3}\left(\left(i \xi+\frac{\kappa}{2}\right) E+A\right)^{-3}\right\|_{H \rightarrow H} \\
& =\sup _{\lambda \in \sigma(A)}\left|\lambda^{3}\left(i \xi+\frac{\kappa}{2}+\lambda\right)^{-3}\right| \\
& =\sup _{\lambda \in \sigma(A)} \frac{\lambda^{3}}{\left((\lambda+\kappa / 2)^{2}+\xi^{2}\right)^{3 / 2}} \leq \sup _{\lambda \in \sigma(A)} \frac{\lambda^{3}}{(\lambda+\kappa / 2)^{3}} \\
& \leq \max \left\{\frac{\lambda_{0}^{3}}{\left(\lambda_{0}+\kappa / 2\right)^{3}}, 1\right\} .
\end{aligned}
$$

Consequently,

$$
\begin{aligned}
& \left\|A^{3}\left(\left(i \xi+\frac{\kappa}{2}\right) E+A\right)^{-3} \hat{g}(\xi)\right\|_{L_{2}(R ; H)} \\
& \quad \leq \max \left\{\frac{\lambda_{0}^{3}}{\left(\lambda_{0}+\kappa / 2\right)^{3}}, 1\right\}\|\hat{g}(\xi)\|_{L_{2}(R ; H)} .
\end{aligned}
$$

Similarly we have

$$
\begin{aligned}
& \left\|-i \xi^{3} \widehat{v}(\xi)\right\|_{L_{2}(R ; H)} \\
& =\left\|-i \xi^{3}\left(\left(i \xi+\frac{\kappa}{2}\right) E+A\right)^{-3} \widehat{g}(\xi)\right\|_{L_{2}(R ; H)} \\
& \quad \leq \sup _{\xi \in R}\left\|-i \xi^{3}\left(\left(i \xi+\frac{\kappa}{2}\right) E+A\right)^{-3}\right\|_{H \rightarrow H} \\
& \cdot\|\widehat{g}(\xi)\|_{L_{2}(R ; H)} .
\end{aligned}
$$

Hence, for $\xi \in R$ and $\kappa>-2 \lambda_{0}$ we obtain

$$
\begin{gathered}
\left\|-i \xi^{3}\left(\left(i \xi+\frac{\kappa}{2}\right) E+A\right)^{-3}\right\|_{H \rightarrow H} \\
=\sup _{\lambda \in \sigma(A)}\left|-i \xi^{3}\left(i \xi+\frac{\kappa}{2}+\lambda\right)^{-3}\right| \\
=\sup _{\lambda \in \sigma(A)} \frac{|\xi|^{3}}{\left((\lambda+\kappa / 2)^{2}+\xi^{2}\right)^{3 / 2}} \\
\leq \frac{|\xi|^{3}}{\left(\left(\lambda_{0}+\kappa / 2\right)^{2}+\xi^{2}\right)^{3 / 2}} \leq 1 .
\end{gathered}
$$


Consequently,

$$
\left\|-i \xi^{3} \widehat{v}(\xi)\right\|_{L_{2}(R ; H)} \leq\|\widehat{g}(\xi)\|_{L_{2}(R ; H)} .
$$

Thus, $v(t) \in W_{2}^{3}(R ; H)$.

It is clear that the vector function $v(t) e^{(\kappa / 2) t}$ belongs to $W_{2, \kappa}^{3}(R ; H)$ and is a regular solution of $(8)$.

It is also clear that the equation $P_{0} u(t)=0$ has only trivial solution in the space $W_{2, \kappa}^{3}(R ; H)$.

Now let us show that $P_{0}$ is a bounded operator from the space $W_{2, \kappa}^{3}(R ; H)$ to the space $L_{2, \kappa}(R ; H)$. In fact, by virtue of Cauchy-Schwarz and Young inequalities, for $u(t) \epsilon$ $W_{2, \kappa}^{3}(R ; H)$, we have

$$
\begin{aligned}
& \operatorname{Re}\left(\frac{d^{3} u}{d t^{3}}, A \frac{d^{2} u}{d t^{2}}\right)_{L_{2, \kappa}(R ; H)} \\
& \leq\left\|\frac{d^{3} u}{d t^{3}}\right\|_{L_{2, \kappa}(R ; H)}\left\|A \frac{d^{2} u}{d t^{2}}\right\|_{L_{2, \kappa}(R ; H)} \\
& \leq \frac{1}{2}\left\|\frac{d^{3} u}{d t^{3}}\right\|_{L_{2, \kappa}(R ; H)}^{2}+\frac{1}{2}\left\|A \frac{d^{2} u}{d t^{2}}\right\|_{L_{2, \kappa}(R ; H)}^{2}, \\
& \operatorname{Re}\left(\frac{d^{3} u}{d t^{3}}, A^{2} \frac{d u}{d t}\right)_{L_{2, \kappa}(R ; H)} \\
& \leq\left\|\frac{d^{3} u}{d t^{3}}\right\|_{L_{2, \kappa}(R ; H)}\left\|A^{2} \frac{d u}{d t}\right\|_{L_{2, \kappa}(R ; H)} \\
& \leq \frac{1}{2}\left\|\frac{d^{3} u}{d t^{3}}\right\|_{L_{2, \kappa}(R ; H)}^{2}+\frac{1}{2}\left\|A^{2} \frac{d u}{d t}\right\|_{L_{2, \kappa}(R ; H)}^{2}, \\
& \operatorname{Re}\left(\frac{d^{3} u}{d t^{3}}, A^{3} u\right)_{L_{2, \kappa}(R ; H)} \\
& \leq\left\|\frac{d^{3} u}{d t^{3}}\right\|_{L_{2, \kappa}(R ; H)}\left\|A^{3} u\right\|_{L_{2, \kappa}(R ; H)} \\
& \leq \frac{1}{2}\left\|\frac{d^{3} u}{d t^{3}}\right\|_{L_{2, \kappa}(R ; H)}^{2}+\frac{1}{2}\left\|A^{3} u\right\|_{L_{2, \kappa}(R ; H)}^{2}, \\
& \operatorname{Re}\left(A \frac{d^{2} u}{d t^{2}}, A^{2} \frac{d u}{d t}\right)_{L_{2, \kappa}(R ; H)} \\
& \leq\left\|A \frac{d^{2} u}{d t^{2}}\right\|_{L_{2, \kappa}(R ; H)}\left\|A^{2} \frac{d u}{d t}\right\|_{L_{2, \kappa}(R ; H)} \\
& \leq \frac{1}{2}\left\|A \frac{d^{2} u}{d t^{2}}\right\|_{L_{2, \kappa}(R ; H)}^{2}+\frac{1}{2}\left\|A^{2} \frac{d u}{d t}\right\|_{L_{2, \kappa}(R ; H)}^{2} \\
& \operatorname{Re}\left(A \frac{d^{2} u}{d t^{2}}, A^{3} u\right)_{L_{2, \kappa}(R ; H)} \\
& \leq\left\|A \frac{d^{2} u}{d t^{2}}\right\|_{L_{2, \kappa}(R ; H)}\left\|A^{3} u\right\|_{L_{2, \kappa}(R ; H)}
\end{aligned}
$$

$$
\begin{aligned}
& \leq \frac{1}{2}\left\|A \frac{d^{2} u}{d t^{2}}\right\|_{L_{2, \kappa}(R ; H)}^{2}+\frac{1}{2}\left\|A^{3} u\right\|_{L_{2, \kappa}(R ; H)}^{2}, \\
\operatorname{Re}\left(A^{2} \frac{d u}{d t}, A^{3} u\right)_{L_{2, \kappa}(R ; H)} & \leq\left\|A^{2} \frac{d u}{d t}\right\|_{L_{2, \kappa}(R ; H)}\left\|A^{3} u\right\|_{L_{2, \kappa}(R ; H)} \\
\leq & \frac{1}{2}\left\|A^{2} \frac{d u}{d t}\right\|_{L_{2, \kappa}(R ; H)}^{2}+\frac{1}{2}\left\|A^{3} u\right\|_{L_{2, \kappa}(R ; H)}^{2} .
\end{aligned}
$$

Then, taking into account intermediate derivatives theorem [2, Chapter 1], we get

$$
\begin{aligned}
& \left\|P_{0} u\right\|_{L_{2, \kappa}(R ; H)}^{2}=\left\|\frac{d^{3} u}{d t^{3}}\right\|_{L_{2, \kappa}(R ; H)}^{2}+9\left\|A \frac{d^{2} u}{d t^{2}}\right\|_{L_{2, \kappa}(R ; H)}^{2} \\
& +9\left\|A^{2} \frac{d u}{d t}\right\|_{L_{2, \kappa}(R ; H)}^{2}+\left\|A^{3} u\right\|_{L_{2, \kappa}(R ; H)}^{2} \\
& +6 \operatorname{Re}\left(\frac{d^{3} u}{d t^{3}}, A \frac{d^{2} u}{d t^{2}}\right)_{L_{2, \kappa}(R ; H)} \\
& +6 \operatorname{Re}\left(\frac{d^{3} u}{d t^{3}}, A^{2} \frac{d u}{d t}\right)_{L_{2, \kappa}(R ; H)} \\
& +2 \operatorname{Re}\left(\frac{d^{3} u}{d t^{3}}, A^{3} u\right)_{L_{2, \kappa}(R ; H)} \\
& +18 \operatorname{Re}\left(A \frac{d^{2} u}{d t^{2}}, A^{2} \frac{d u}{d t}\right)_{L_{2, \kappa}(R ; H)} \\
& +6 \operatorname{Re}\left(A \frac{d^{2} u}{d t^{2}}, A^{3} u\right)_{L_{2, \kappa}(R ; H)} \\
& +6 \operatorname{Re}\left(A^{2} \frac{d u}{d t}, A^{3} u\right)_{L_{2, \kappa}(R ; H)} \leq 8\left\|\frac{d^{3} u}{d t^{3}}\right\|_{L_{2, \kappa}(R ; H)}^{2} \\
& +24\left\|A \frac{d^{2} u}{d t^{2}}\right\|_{L_{2, \kappa}(R ; H)}^{2}+24\left\|A^{2} \frac{d u}{d t}\right\|_{L_{2, \kappa}(R ; H)}^{2} \\
& +8\left\|A^{3} u\right\|_{L_{2, \kappa}(R ; H)}^{2}=8\left(\|u\|_{W_{2, \kappa}^{3}(R ; H)}^{2}\right. \\
& \left.+3\left\|A \frac{d^{2} u}{d t^{2}}\right\|_{L_{2, \kappa}(R ; H)}^{2}+3\left\|A^{2} \frac{d u}{d t}\right\|_{L_{2, \kappa}(R ; H)}^{2}\right) \\
& \leq \text { const }\|u\|_{W_{2, \kappa}^{3}(R ; H)}^{2} ;
\end{aligned}
$$

that is,

$$
\left\|P_{0} u\right\|_{L_{2, \kappa}(R ; H)} \leq \text { const }\|u\|_{W_{2, \kappa}^{3}(R ; H)} .
$$


So we obtain that the operator $P_{0}: W_{2, \kappa}^{3}(R ; H) \rightarrow$ $L_{2, \kappa}(R ; H)$ is one-to-one and bounded. Then, by the Banach theorem on inverse operator, the operator $P_{0}^{-1}: L_{2, \kappa}(R ; H) \rightarrow$ $W_{2, \kappa}^{3}(R ; H)$ is bounded.

Thus, $P_{0}$ is an isomorphism between the spaces $W_{2, \kappa}^{3}(R ; H)$ and $L_{2, \kappa}(R ; H)$. Theorem is proved.

Theorem 2 has the following.

Corollary 3. When $\kappa>-2 \lambda_{0}$, the norms $\left\|P_{0} u\right\|_{L_{2, \kappa}(R ; H)}$ and $\|u\|_{W_{2, \kappa}^{3}(R ; H)}$ are equivalent in the space $W_{2, \kappa}^{3}(R ; H)$.

Remark 4. For $\kappa=-2 \lambda_{0}$, the operator $P_{0}$ is noninvertible.

As is known, the intermediate derivative operators

$$
A^{j} \frac{d^{3-j}}{d t^{3-j}}: W_{2, \kappa}^{3}(R ; H) \longrightarrow L_{2, \kappa}(R ; H), \quad j=1,2,
$$

are continuous [2]. By virtue of this fact and Corollary 3, the norms of the operators (30) can be estimated through $\left\|P_{0} u\right\|_{L_{2, \kappa}(R ; H)}$.

Theorem 5. Let $A$ be a self-adjoint positive definite operator with the lower bound for its spectrum $\lambda_{0}\left(A=A^{*} \geq\right.$ $\lambda_{0} E, \lambda_{0}>0$ ) and $\kappa>-2 \lambda_{0}$. Then, for every $u(t) \epsilon$ $W_{2, \kappa}^{3}(R ; H)$ the following inequalities are true:

$$
\left\|A^{j} \frac{d^{3-j} u}{d t^{3-j}}\right\|_{L_{2, \kappa}(R ; H)} \leq c_{j}\left(\kappa ; \lambda_{0}\right)\left\|P_{0} u\right\|_{L_{2, \kappa}(R ; H)},
$$

$$
j=1,2 \text {, }
$$

where

$$
\begin{aligned}
& c_{1}\left(\kappa ; \lambda_{0}\right)= \begin{cases}\frac{2 \lambda_{0} \kappa^{2}}{\left(2 \lambda_{0}+\kappa\right)^{3}}, & -2 \lambda_{0}<\kappa \leq-\lambda_{0}, \\
\frac{2}{3^{3 / 2}\left(1+\kappa / \lambda_{0}\right)^{1 / 2}}, & \kappa>-\lambda_{0},\end{cases} \\
& c_{2}\left(\kappa ; \lambda_{0}\right)= \begin{cases}\frac{4 \lambda_{0}^{2}|\kappa|}{\left(2 \lambda_{0}+\kappa\right)^{3}}, & -2 \lambda_{0}<\kappa \leq-\lambda_{0}, \\
\frac{2}{3^{3 / 2}\left(1+\kappa / \lambda_{0}\right)}, & \kappa>-\lambda_{0} .\end{cases}
\end{aligned}
$$

Proof. As the mapping $v(t) \rightarrow u(t) e^{-(\kappa / 2) t}$ is an isomorphism between the spaces $W_{2}^{3}(R ; H)$ and $W_{2, \kappa}^{3}(R ; H)$, to prove inequalities (31) it suffices to estimate the norms $\| A^{j}(d / d t+$ $\kappa / 2)^{3-j} v \|_{L_{2}(R ; H)}, j=1,2$, by $\left\|P_{0, \kappa} v\right\|_{L_{2}(R ; H)}$. Then, making a change $P_{0, \kappa} v(t)=g(t)$ and applying Fourier transform, we obtain

$$
\begin{aligned}
& \left\|A^{j}\left(i \xi+\frac{\kappa}{2}\right)^{3-j} P_{0, \kappa}^{-1}(i \xi ; A) \hat{g}(\xi)\right\|_{L_{2}(R ; H)} \\
& \quad \leq \sup _{\xi \in R}\left\|A^{j}\left(i \xi+\frac{\kappa}{2}\right)^{3-j}\left(\left(i \xi+\frac{\kappa}{2}\right) E+A\right)^{-3}\right\|_{H \rightarrow H} \\
& \cdot\|\widehat{g}(\xi)\|_{L_{2}(R ; H)}, \quad j=1,2 .
\end{aligned}
$$

Consequently, for $\xi \in R$ and $\kappa>-2 \lambda_{0}$, we need to estimate the norms

$$
\begin{gathered}
\left\|A^{j}\left(i \xi+\frac{\kappa}{2}\right)^{3-j}\left(\left(i \xi+\frac{\kappa}{2}\right) E+A\right)^{-3}\right\|_{H \rightarrow H} \\
=\sup _{\lambda \in \sigma(A)}\left|\frac{\lambda^{j}(i \xi+\kappa / 2)^{3-j}}{(i \xi+\kappa / 2+\lambda)^{3}}\right| \\
=\sup _{\lambda \in \sigma(A)} \frac{\lambda^{j}\left(\xi^{2}+\kappa^{2} / 4\right)^{(3-j) / 2}}{\left((\lambda+\kappa / 2)^{2}+\xi^{2}\right)^{3 / 2}}, \quad j=1,2 .
\end{gathered}
$$

Consider the case $j=1$. Solving extremal problem, we have

$$
\begin{aligned}
& \sup _{\lambda \in \sigma(A)} \frac{\lambda\left(\xi^{2}+\kappa^{2} / 4\right)}{\left((\lambda+\kappa / 2)^{2}+\xi^{2}\right)^{3 / 2}} \\
& \leq \sup _{\lambda \geq \lambda_{0}, \xi \in R} \frac{\xi^{2} / \lambda^{2}+\kappa^{2} /\left(4 \lambda_{0}^{2}\right)}{\left(\left(1+\kappa /\left(2 \lambda_{0}\right)\right)^{2}+\xi^{2} / \lambda^{2}\right)^{3 / 2}} \\
& =\sup _{\xi^{2} / \lambda^{2} \geq 0} \frac{\xi^{2} / \lambda^{2}+\kappa^{2} /\left(4 \lambda_{0}^{2}\right)}{\left(\left(1+\kappa /\left(2 \lambda_{0}\right)\right)^{2}+\xi^{2} / \lambda^{2}\right)^{3 / 2}}=c_{1}\left(\kappa ; \lambda_{0}\right),
\end{aligned}
$$

where $c_{1}\left(\kappa ; \lambda_{0}\right)=2 \lambda_{0} \kappa^{2} /\left(2 \lambda_{0}+\kappa\right)^{3}$ if $-2 \lambda_{0}<\kappa \leq-\lambda_{0}$, and $c_{1}\left(\kappa ; \lambda_{0}\right)=2 /\left(3^{3 / 2}\left(1+\kappa / \lambda_{0}\right)^{1 / 2}\right)$ if $\kappa>-\lambda_{0}$.

For $j=2$, we have

$$
\sup _{\lambda \in \sigma(A)} \frac{\lambda^{2}\left(\xi^{2}+\kappa^{2} / 4\right)^{1 / 2}}{\left((\lambda+\kappa / 2)^{2}+\xi^{2}\right)^{3 / 2}}
$$

$$
\begin{aligned}
& \leq \sup _{\lambda \geq \lambda_{0}, \xi \in R} \frac{\left(\xi^{2} / \lambda^{2}+\kappa^{2} /\left(4 \lambda_{0}^{2}\right)\right)^{1 / 2}}{\left(\left(1+\kappa /\left(2 \lambda_{0}\right)\right)^{2}+\xi^{2} / \lambda^{2}\right)^{3 / 2}} \\
& =\sup _{\xi^{2} / \lambda^{2} \geq 0} \frac{\left(\xi^{2} / \lambda^{2}+\kappa^{2} /\left(4 \lambda_{0}^{2}\right)\right)^{1 / 2}}{\left(\left(1+\kappa /\left(2 \lambda_{0}\right)\right)^{2}+\xi^{2} / \lambda^{2}\right)^{3 / 2}} \\
& =c_{2}\left(\kappa ; \lambda_{0}\right),
\end{aligned}
$$

where $c_{2}\left(\kappa ; \lambda_{0}\right)=4 \lambda_{0}^{2}|\kappa| /\left(2 \lambda_{0}+\kappa\right)^{3}$ if $-2 \lambda_{0}<\kappa \leq-\lambda_{0}$, and $c_{2}\left(\kappa ; \lambda_{0}\right)=2 /\left(3^{3 / 2}\left(1+\kappa / \lambda_{0}\right)\right)$ if $\kappa>-\lambda_{0}$.

Thus, considering the obtained estimates in inequalities (33), we have

$$
\begin{gathered}
\left\|A^{j}\left(i \xi+\frac{\kappa}{2}\right)^{3-j} P_{0, \kappa}^{-1}(i \xi ; A) \widehat{g}(\xi)\right\|_{L_{2}(R ; H)} \\
\quad \leq c_{j}\left(\kappa ; \lambda_{0}\right)\|\widehat{g}(\xi)\|_{L_{2}(R ; H)}, \quad j=1,2 .
\end{gathered}
$$

The latter inequalities, in turn, are equivalent to the following ones:

$$
\begin{array}{r}
\left\|A^{j}\left(\frac{d}{d t}+\frac{\kappa}{2}\right)^{3-j} v\right\|_{L_{2}(R ; H)} \leq c_{j}\left(\kappa ; \lambda_{0}\right)\left\|P_{0, \kappa} v\right\|_{L_{2}(R ; H)}, \\
j=1,2 .
\end{array}
$$

Theorem is proved. 


\section{Solvability of (5) in $W_{2, \kappa}^{3}(R ; H)$}

When $A_{j} \neq 0, j=1,2$

Denote by $P_{1}$ the operator acting from $W_{2, \kappa}^{3}(R ; H)$ to $L_{2, \kappa}(R ; H)$ as follows:

$$
\begin{aligned}
& P_{1} u(t) \equiv A_{1} \frac{d^{2} u(t)}{d t^{2}}+A_{2} \frac{d u(t)}{d t}, \\
& u(t) \in W_{2, \kappa}^{3}(R ; H) .
\end{aligned}
$$

The following lemma is true.

Lemma 6. Let $A$ be a self-adjoint positive definite operator in $H$ and let $A_{j} A^{-j} \in L(H), j=1,2$. Then the operator $P_{1}$ is bounded from $W_{2, \kappa}^{3}(R ; H)$ to $L_{2, \kappa}(R ; H)$.

Proof. By the conditions of lemma, for $u(t) \in W_{2, \kappa}^{3}(R ; H)$, we have

$$
\begin{aligned}
\left\|P_{1} u\right\|_{L_{2, \kappa}(R ; H)}= & \left\|A_{1} \frac{d^{2} u}{d t^{2}}+A_{2} \frac{d u}{d t}\right\|_{L_{2, \kappa}(R ; H)} \\
\leq & \left\|A_{1} \frac{d^{2} u}{d t^{2}}\right\|_{L_{2, \kappa}(R ; H)}+\left\|A_{2} \frac{d u}{d t}\right\|_{L_{2, \kappa}(R ; H)} \\
\leq & \left\|A_{1} A^{-1}\right\|_{H \rightarrow H}\left\|A \frac{d^{2} u}{d t^{2}}\right\|_{L_{2, \kappa}(R ; H)} \\
& +\left\|A_{2} A^{-2}\right\|_{H \rightarrow H}\left\|A^{2} \frac{d u}{d t}\right\|_{L_{2, \kappa}(R ; H)} .
\end{aligned}
$$

Using intermediate derivatives theorem [2, Chapter 1], we get

$$
\left\|P_{1} u\right\|_{L_{2, \kappa}(R ; H)} \leq \text { const }\|u\|_{W_{2, \kappa}^{3}(R ; H)} .
$$

Lemma is proved.

The obtained results allow studying (5) in case $A_{j} \neq 0$, $j=1,2$.

Denote by $P$ the operator which acts from $W_{2, \kappa}^{3}(R ; H)$ to $L_{2, \kappa}(R ; H)$ as follows:

$$
P u(t)=P_{0} u(t)+P_{1} u(t), \quad u(t) \in W_{2, \kappa}^{3}(R ; H) .
$$

The lemma below can be proved with the help of Theorem 2 and Lemma 6.

Lemma 7. Let $A$ be a self-adjoint positive definite operator in $H$ and let $A_{j} A^{-j} \in L(H), j=1,2$. Then the operator $P$ acts boundedly from $W_{2, \kappa}^{3}(R ; H)$ to $L_{2, \kappa}(R ; H)$.

Now let us state the main result of this work.

Theorem 8. Let $A=A^{*} \geq \lambda_{0} E, \lambda_{0}>0, \kappa>-2 \lambda_{0}$, and $A_{j} A^{-j} \in L(H), j=1,2$, and let the inequality

$$
\begin{aligned}
& c_{1}\left(\kappa ; \lambda_{0}\right)\left\|A_{1} A^{-1}\right\|_{H \rightarrow H}+c_{2}\left(\kappa ; \lambda_{0}\right)\left\|A_{2} A^{-2}\right\|_{H \rightarrow H} \\
& \quad<1
\end{aligned}
$$

be true, where the numbers $c_{1}\left(\kappa ; \lambda_{0}\right)$ and $c_{2}\left(\kappa ; \lambda_{0}\right)$ are defined as in Theorem 5. Then (5) is regularly solvable.
Proof. Let us write (5) in the form of operator equation:

$$
P_{0} u(t)+P_{1} u(t)=f(t),
$$

where $f(t) \in L_{2, \kappa}(R ; H), u(t) \in W_{2, \kappa}^{3}(R ; H)$.

By Theorem 2, (8) is regularly solvable. Make a change $P_{0} u(t)=w(t)$ and rewrite (44) as follows:

$$
\left(E+P_{1} P_{0}^{-1}\right) w(t)=f(t) .
$$

By Theorem 5 , for every $w(t) \in L_{2, \kappa}(R ; H)$, we have

$$
\begin{aligned}
& \left\|P_{1} P_{0}^{-1} w\right\|_{L_{2, \kappa}(R ; H)}=\left\|P_{1} u\right\|_{L_{2, \kappa}(R ; H)} \leq\left\|A_{1} A^{-1}\right\|_{H \rightarrow H} \\
& .\left\|A \frac{d^{2} u}{d t^{2}}\right\|_{L_{2, \kappa}(R ; H)}+\left\|A_{2} A^{-2}\right\|_{H \rightarrow H}\left\|A^{2} \frac{d u}{d t}\right\|_{L_{2, \kappa}(R ; H)} \\
& \leq\left\|A_{1} A^{-1}\right\|_{H \rightarrow H} c_{1}\left(\kappa ; \lambda_{0}\right)\left\|P_{0} u\right\|_{L_{2, \kappa}(R ; H)} \\
& +\left\|A_{2} A^{-2}\right\|_{H \rightarrow H} c_{2}\left(\kappa ; \lambda_{0}\right)\left\|P_{0} u\right\|_{L_{2, \kappa}(R ; H)} \\
& =\left(c_{1}\left(\kappa ; \lambda_{0}\right)\left\|A_{1} A^{-1}\right\|_{H \rightarrow H}\right. \\
& \left.\quad+c_{2}\left(\kappa ; \lambda_{0}\right)\left\|A_{2} A^{-2}\right\|_{H \rightarrow H}\right)\|w\|_{L_{2, \kappa}(R ; H)} .
\end{aligned}
$$

As the operator $E+P_{1} P_{0}^{-1}$ is invertible in the space $L_{2, \kappa}(R ; H)$, when

$$
\begin{aligned}
& c_{1}\left(\kappa ; \lambda_{0}\right)\left\|A_{1} A^{-1}\right\|_{H \rightarrow H}+c_{2}\left(\kappa ; \lambda_{0}\right)\left\|A_{2} A^{-2}\right\|_{H \rightarrow H} \\
& \quad<1,
\end{aligned}
$$

we can define $u(t)$ by the formula

$$
u(t)=P_{0}^{-1}\left(E+P_{1} P_{0}^{-1}\right)^{-1} f(t),
$$

and we have

$$
\begin{aligned}
& \|u\|_{W_{2, \kappa}^{3}(R ; H)} \leq\left\|P_{0}^{-1}\right\|_{L_{2, \kappa}(R ; H) \rightarrow W_{2, \kappa}^{3}(R ; H)} \\
& \quad\left\|\left(E+P_{1} P_{0}^{-1}\right)^{-1}\right\|_{L_{2, \kappa}(R ; H) \rightarrow L_{2, \kappa}(R ; H)}\|f\|_{L_{2, \kappa}(R ; H)} \\
& \quad \leq \text { const }\|f\|_{L_{2, \kappa}(R ; H)} .
\end{aligned}
$$

Theorem is proved.

Theorem 8 has the following.

Corollary 9. Under the conditions of Theorem 8, the operator $P$ is an isomorphism between the spaces $W_{2, \kappa}^{3}(R ; H)$ and $L_{2, \kappa}(R ; H)$.

In conclusion, let us illustrate our main result by the example of a problem for a partial differential equation. Consider the following problem on the strip $R \times[0, \pi]$ :

$$
\begin{aligned}
& \left(\frac{\partial}{\partial t}-\frac{\partial^{2}}{\partial x^{2}}\right)^{3} u(t, x)+p(x) \frac{\partial^{4} u(t, x)}{\partial x^{2} \partial t^{2}} \\
& \quad+q(x) \frac{\partial^{5} u(t, x)}{\partial x^{4} \partial t}=f(t, x), \\
& \frac{\partial^{2 s} u(t, 0)}{\partial x^{2 s}}=\frac{\partial^{2 s} u(t, \pi)}{\partial x^{2 s}}=0, \quad s=0,1,2,
\end{aligned}
$$


where $f(t, x) \in L_{2, \kappa}\left(R ; L_{2}[0, \pi]\right)$, and $p(x), q(x)$ are the functions bounded on $[0, \pi]$. The problem $(50)$ can be easily reduced to (5), with $H=L_{2}[0, \pi], A_{1}=p(x)\left(\partial^{2} / \partial x^{2}\right)$, $A_{2}=q(x)\left(\partial^{4} / \partial x^{4}\right)$, and the operator $A$ being defined in $L_{2}[0, \pi]$ by the equality $A u=-d^{2} u / d x^{2}$ and the conditions $u(0)=u(\pi)=0$. In this case, $\lambda_{0}=1$. Applying Theorem 8 , we obtain that if $\kappa>-2$ and

$$
c_{1}(\kappa ; 1) \sup _{x \in[0, \pi]}|p(x)|+c_{2}(\kappa ; 1) \sup _{x \in[0, \pi]}|q(x)|<1,
$$

where

$$
\begin{gathered}
c_{1}(\kappa ; 1)= \begin{cases}\frac{2 \kappa^{2}}{(2+\kappa)^{3}}, & -2<\kappa \leq-1, \\
\frac{2}{3^{3 / 2}(1+\kappa)^{1 / 2}}, & \kappa>-1,\end{cases} \\
c_{2}\left(\kappa ; \lambda_{0}\right)= \begin{cases}\frac{4|\kappa|}{(2+\kappa)^{3}}, & -2<\kappa \leq-1, \\
\frac{2}{3^{3 / 2}(1+\kappa)}, & \kappa>-1,\end{cases}
\end{gathered}
$$

then problem (50) has a unique solution in the space $W_{t, x, 2, \kappa}^{3,6}\left(R ; L_{2}[0, \pi]\right)$.

\section{Conflicts of Interest}

The authors declare that there are no conflicts of interest regarding the publication of this paper.

\section{References}

[1] E. Hille and R. S. Phillips, Functional analysis and semi-groups, American Mathematical Society Colloquium Publications, vol. 31, American Mathematical Society, Providence, RI, USA, 1957.

[2] J. L. Lions and E. Magenes, Non-Homogeneous Boundary Value Problems and Applications, Springer, Berlin, Germany, 1972.

[3] Ju. A. Dubinskiī, "On some differential-operator equations of arbitrary order," Mathematics of the USSR-Sbornik, vol. 19, no. 1, pp. 1-21, 1973, (published in Matematicheskii Sbornik, vol. 90 (132), no. 1, pp. 3-22, 1973).

[4] M. H. Ilyasov, "Mixed problems for a class of pseudoparabolic equations," Indian Journal of Pure and Applied Mathematics, vol. 38, no. 2, pp. 97-103, 2007.

[5] S. P. Kapitza, "A mathematical model for global population growth," Matematicheskoe Modelirovanie, vol. 4, no. 6, pp. 6579, 1992 (Russian).

[6] S. G. Krein, Linear Differential Equations in a Banach Space, Nauka, Moscow, Russia, 1967.

[7] V. I. Gorbachuk and M. L. Gorbachuk, Boundary Value Problems for Operator-Differential Equations, Naukova Dumka, Kiev, Ukraine, 1984.

[8] J. A. Goldstein, Semigroups of Linear Operators and Applications, Oxford Mathematical Monographs, The Clarendon Press, Oxford University Press, New York, NY, USA, 1985.

[9] S. Ya. Yakubov, Linear Differential-Operator Equations and their Applications, Elm, Baku, Azerbaijan, 1985.

[10] S. Yakubov, Completeness of Root Functions of Regular Differential Operators, vol. 71 of Pitman Monographs and Surveys in Pure and Applied Mathematics, Longman, Scientific and Technical, New York, NY, USA, 1994.

[11] S. Yakubov and Y. Yakubov, Differential-Operator Equations, vol. 103 of Chapman \& Hall/CRC Monographs and Surveys in Pure and Applied Mathematics, Chapman and Hall/CRC, Boca Raton, Fla, USA, 2000.

[12] M. G. Gasymov, "On the theory of polynomial operator pencils," Soviet Mathematics - Doklady, vol. 12, pp. 1143-1147, 1972, (published in Doklady Akademii Nauk SSSR, vol. 199, no. 4, pp. 747-750, 1971).

[13] M. G. Gasymov, "The multiple completeness of part of the eigen- and associated vectors of polynomial operator bundles," Izvestija Akademii Nauk Armjanskoui SSR. Serija Matematika, vol. 6, no. 2-3, pp. 131-147, 1971 (Russian).

[14] M. G. Gasymov, "On the solubility of boundary value problems for a class of operator-differential equations," Soviet Mathematics - Doklady, vol. 18, pp. 943-947, 1977, (published in Doklady Akademii Nauk SSSR, vol. 235, no. 3, pp. 505-508, 1977).

[15] A. R. Aliev, "On the solvability of initial-boundary value problems for a class of third-order operator-differential equations," Mathematical Notes, vol. 90, no. 3, pp. 307-321, 2011, (published in Matematicheskie Zametki, vol. 90, no. 3, pp. 323-339, 2011).

[16] A. R. Aliev and S. S. Mirzoev, "On the theory of the solvability of boundary value problems for a class of higher-order operatordifferential equations," Functional Analysis and Its Applications, vol. 44, no. 3, pp. 209-211, 2010, (published in Funktsional'nyi Analiz i ego Prilozheniya, vol. 44, no. 3, pp. 63-65, 2010).

[17] A. Favini, V. Shakhmurov, and Y. Yakubov, "Regular boundary value problems for complete second order elliptic differentialoperator equations in UMD Banach spaces," Semigroup Forum, vol. 79, no. 1, pp. 22-54, 2009.

[18] A. Favini and Y. Yakubov, "Higher order ordinary differentialoperator equations on the whole axis in UMD Banach spaces," Differential and Integral Equations, vol. 21, no. 5-6, pp. 497-512, 2008.

[19] A. Favini and Y. Yakubov, "Irregular boundary value problems for second order elliptic differential-operator equations in UMD Banach spaces," Mathematische Annalen, vol. 348, no. 3, pp. 601-632, 2010.

[20] A. Favini, R. Labbas, K. Lemrabet, S. Maingot, and H. D. Sidibé, "Transmission problem for an abstract fourth-order differential equation of elliptic type in UMD spaces," Advances in Differential Equations, vol. 15, no. 1-2, pp. 43-72, 2010.

[21] S. S. Mirzoev, A. R. Aliev, and L. A. Rustamova, "Solvability conditions for boundary-value problems for elliptic operatordifferential equations with discontinuous coefficient," Mathematical Notes, vol. 92, no. 5-6, pp. 722-726, 2012, (published in Matematicheskie Zametki, vol. 92, no. 5, pp. 789-793, 2012).

[22] S. S. Mirzoev, A. R. Aliev, and L. A. Rustamova, "On the boundary value problem with the operator in boundary conditions for the operator-differential equation of second order with discontinuous coefficients," Journal of Mathematical Physics, Analysis, Geometry, vol. 9, no. 2, pp. 207-226, 2013.

[23] B. A. Aliev and Y. Yakubov, "Fredholm property of boundary value problems for a fourth-order elliptic differentialoperator equation with operator boundary conditions," Differential Equations, vol. 50, no. 2, pp. 213-219, 2014, (published in Differentsial'nye Uravneniya, vol. 50, no. 2, pp. 210-216, 2014).

[24] B. A. Aliev and Y. Yakubov, "Solvability of boundary value problems for second-order elliptic differential-operator equations with a spectral parameter and with a discontinuous coefficient 
at the highest derivative," Differential Equations, vol. 50, no. 4, pp. 464-475, 2014, (published in Differentsial'nye Uravneniya, vol. 50, no. 4, pp. 468-479, 2014).

[25] S. S. Mirzoev, A. R. Aliev, and G. M. Gasimova, "Solvability conditions of a boundary value problem with operator coefficients and related estimates of the norms of intermediate derivative operators," Doklady Mathematics, vol. 94, no. 2, pp. 566-568, 2016, (published in Doklady Akademii Nauk, vol. 470, no. 5, pp. 511-513, 2016).

[26] A. A. Shkalikov, "Elliptic equations in a Hilbert space and related spectral problems," Journal of Soviet Mathematics, vol. 51, no. 4, pp. 2399-2467, 1990, (published in Trudy Seminara imeni I. G. Petrovskogo, no. 14, pp. 140-224, 1989).

[27] S. S. Mirzoev, "On solvability of boundary-value problems for operator-differential equations of the second order in spaces with weight," in Linear Operators and Their Applications, Baku, Azerbaijan, pp. 46-49, 1989.

[28] A. R. Aliev, "On the solvability of a boundary value problem for a class of third-order operator-differential equations in a weighted space," Russian Mathematical Surveys, vol. 60, no. 4, pp. 791-793, 2005, (published in Uspekhi Matematicheskikh Nauk, vol. 60, no. 4 (364), pp. 215-216, 2005).

[29] A. R. Aliev, "On the solvability of a fourth-order operatordifferential equation with multiple characteristic," Ukrainian Mathematical Journal, vol. 66, no. 5, pp. 781-791, 2014, (published in Ukrains'kyi Matematychnyi Zhurnal, vol. 66, no. 5, pp. 699-707, 2014).

[30] S. S. Mirzoev and R. Z. Humbataliev, "On normal solvability of boundary value problems for operator-differential equations on semi-axis in weight space," Taiwanese Journal of Mathematics, vol. 15, no. 4, pp. 1637-1650, 2011.

[31] A. R. Aliev and A. L. Elbably, "On the solvability in a weighted space of a third-order operator-differential equation with multiple characteristics," Doklady Mathematics, vol. 85, no. 2, pp. 233-235, 2012, (published in Doklady Akademii Nauk, vol. 443, no. 4, pp. 407-409, 2012).

[32] A. R. Aliev and F. S. Lachinova, "On the solvability in a weighted space of an initial-boundary value problem for a third-order operator-differential equation with a parabolic principal part," Doklady Mathematics, vol. 93, no. 1, pp. 85-88, 2016, (published in Doklady Akademii Nauk, vol. 466, no. 6, pp. 637-640, 2016).

[33] V. I. Burenkov, "Exact constants in inequalities for norms of intermediate derivatives on a finite interval," Akademiya Nauk Soyuza Sovetskikh Sotsialisticheskikh Respublik. Trudy Matematicheskogo Instituta imeni V. A. Steklova, vol. 156, pp. 23-30, 1983, (published in Investigations in the theory of differentiable functions of many variables and its applications. Part 8, Work collection, Trudy Matematicheskogo Instituta imeni V. A. Steklova, vol. 156, pp. 22-29, 1980).

[34] G. G. Magaril-Il'yaev and V. M. Tikhomirov, "Kolmogorov-type inequalities for derivatives," Sbornik: Mathematics, vol. 188, no. 12, pp. 1799-1832, 1997, (published in Matematicheskii Sbornik, vol. 188, no. 12, pp. 73-106, 1997). 


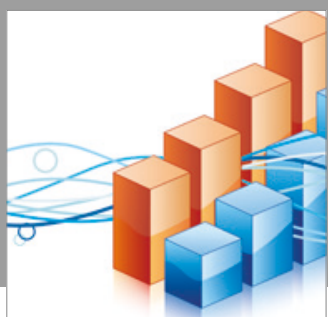

Advances in

Operations Research

vatersals

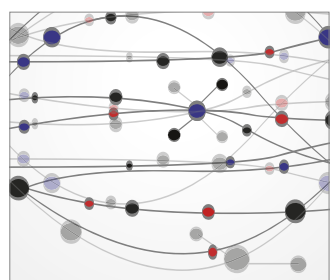

\section{The Scientific} World Journal
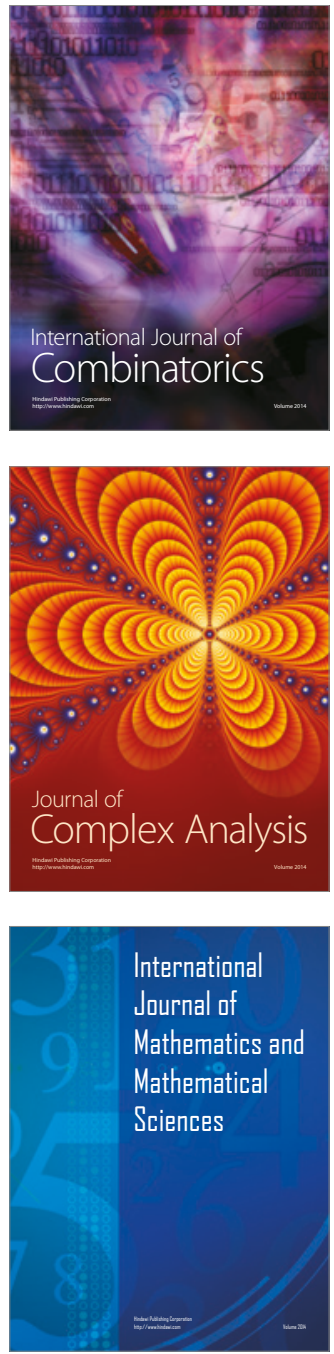
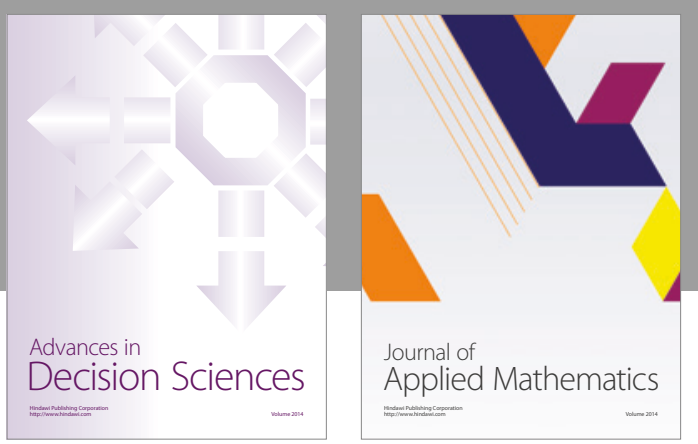

Algebra

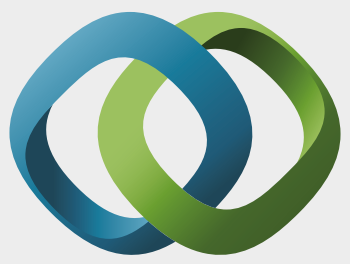

\section{Hindawi}

Submit your manuscripts at

https://www.hindawi.com
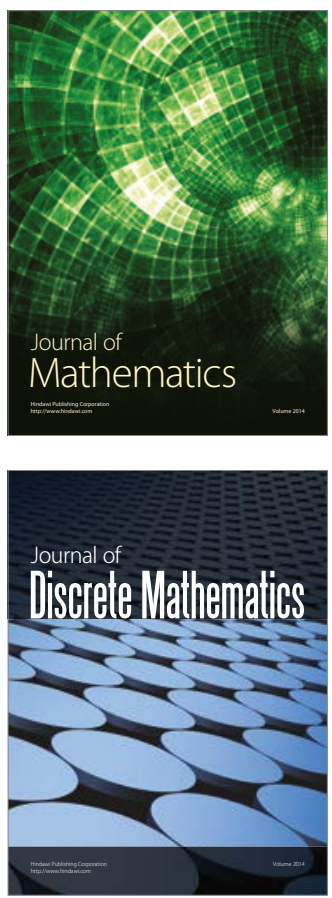

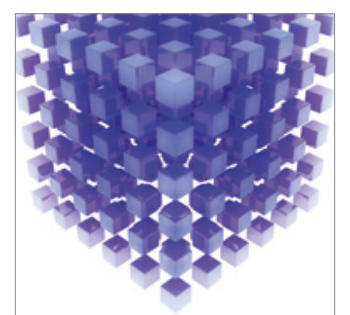

Mathematical Problems in Engineering
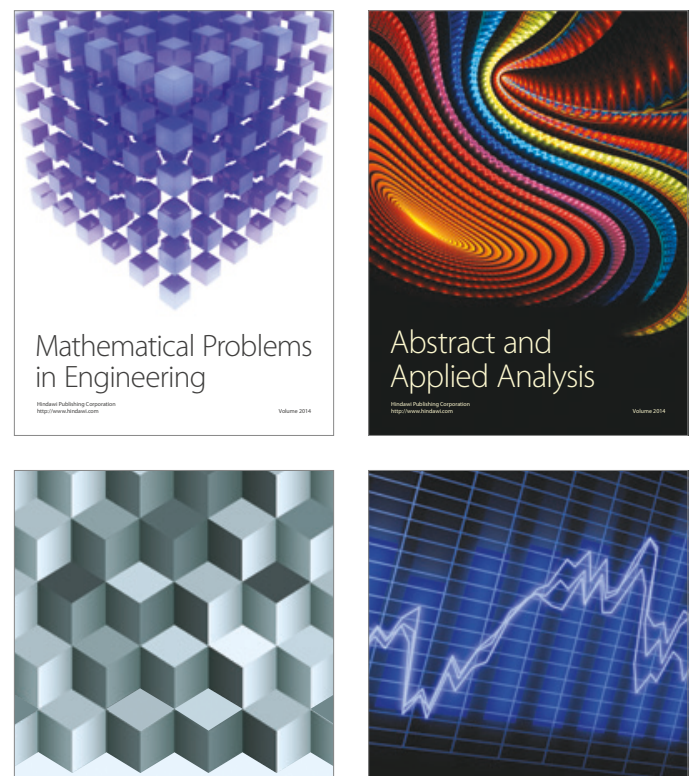

Journal of

Function Spaces

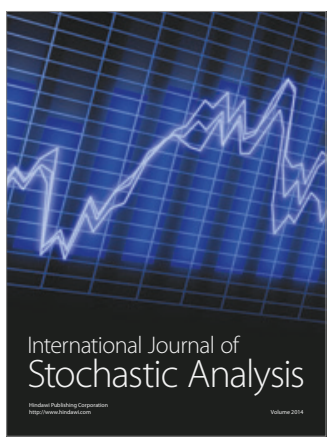

Probability and Statistics
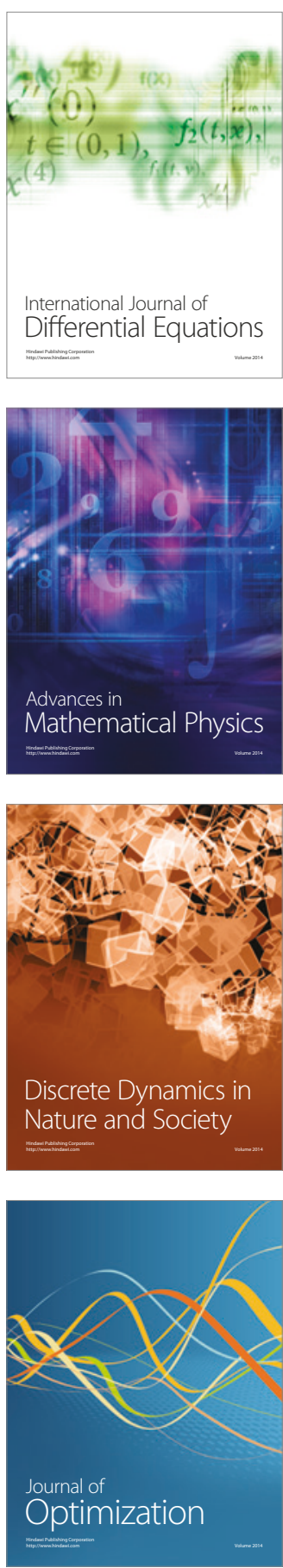